\title{
Branching-annihilating random walks in one dimension: Some exact results
}

\author{
K. Mussawisade ${ }^{1}$, J. E. Santos ${ }^{2}$ and G. M. Schütz ${ }^{1}$ \\ ${ }^{1}$ Institut für Festkörperforschung, Forschungszentrum Jülich, 52425 Jülich, Germany \\ ${ }^{2}$ Department of Theoretical Physics, University of Oxford, 1 Keble Road, Oxford, OX1 3NP, United Kingdom
}

(August 13, 2018)

\begin{abstract}
We derive a self-duality relation for a one-dimensional model of branching and annihilating random walkers with an even number of offsprings. With the duality relation and by deriving exact results in some limiting cases involving fast diffusion we obtain new information on the location and nature of the phase transition line between an active stationary state (non-zero density) and an absorbing state (extinction of all particles), thus clarifying some so far open problems. In these limits the transition is mean-field-like, but on the active side of the phase transition line the fluctuation in the number of particles deviates from its mean-field value. We also show that well within the active region of the phase diagram a finite system approaches the absorbing state very slowly on a time scale which diverges exponentially in system size.

PACS numbers: 05.70.Ln, 05.40.+j, 64.60.Ht, 02.50.-r
\end{abstract}

\section{INTRODUCTION}

In a branching-annihilating random walk (BARW) particles hop on a lattice, annihilate pairwise on encounter, but may also spontaneously create offsprings on the same or on nearest neighbour lattice sites. Such models appear in a large variety of contexts, in particular in reaction-diffusion mechanisms and in non-equilibrium spin relaxation. A generic feature of these processes is a transition as a function of the annihilation and branching rates between an active stationary state with non-zero particle density and an absorbing, inactive state in which all particles are extinct. Numerical results gained from a large variety of systems suggest that the transition in models with a single (or an odd number) of offsprings fall into the universality class of directed percolation (DP) [1], whereas models with an even number of offsprings belong to a distinct parity-conserving (PC) universality class [2, 3]. A coherent picture of this scenario is provided from an renormalization point of view 画.

In this paper we use exact methods to derive a selfduality relation and to address some open questions for limiting cases of the BARW model of Ref. [3] which is a model for spin relaxation dynamics far from thermal equilibrium. One considers Ising spins in one dimension with generalized zero-temperature Glauber dynamics [5], but with an independent coupling to an infinitetemperature heat bath which allows for Kawasaki spinexchange events [6] with rate $\alpha / 2$. This spin-flip process can be visualized in the following way:

$$
\begin{aligned}
& \uparrow \downarrow \uparrow \rightarrow \uparrow \uparrow \uparrow \text { and } \downarrow \uparrow \downarrow \rightarrow \downarrow \downarrow \downarrow \text { with rate } \lambda \\
& \uparrow \uparrow \downarrow \rightleftharpoons \uparrow \downarrow \downarrow \text { and } \downarrow \downarrow \uparrow \rightleftharpoons \downarrow \uparrow \uparrow \text { with rate } D / 2 \\
& \\
& \quad \uparrow \downarrow \rightleftharpoons \downarrow \uparrow \text { and } \downarrow \uparrow \uparrow \rightleftharpoons \uparrow \downarrow \text { with rate } \alpha / 2
\end{aligned}
$$

By identifying a domain wall ( $\downarrow \downarrow$ or $\downarrow \uparrow)$ with a particle of type $A$ on the dual lattice and two parallel spins with a vacancy $\emptyset[7]$ this process becomes a BARW with rates

$$
\begin{gathered}
A A \rightarrow \emptyset \emptyset \text { with rate } \lambda \\
\emptyset A \rightleftharpoons A \emptyset \text { with rate } D / 2 \\
\emptyset A \emptyset \rightleftharpoons A A A \text { with rate } \alpha / 2 \\
\emptyset A A \rightleftharpoons A A \emptyset \text { with rate } \alpha / 2
\end{gathered}
$$

Without branching (i.e. spin exchange) the system evolves into the single absorbing state with no particles at all. In spin language this is the totally ferromagnetic state with all spins up or all spins down. In the presence of the branching process an intricate competition between the zero-temperature ordering process (particle annihilation) and the disordering high-temperature branching process sets in. The result is a non-trivial phase diagram as a function of the system parameters. Starting from, say, a random initial state with an even number of particles the system evolves ultimately into the inactive empty lattice for dominant ordering dynamics, whereas it remains in an active state with finite density if the disordering branching process dominates. Numerical evidence suggests the phase transition to belong to the PC universality class [3].

We stress that these results are supposed to be valid only in the thermodynamic limit. In any finite system 
the unique stationary state is the absorbing inactive state (unless $\lambda=0$ ) because for $\lambda>0$ there is always a small probability of reaching this state from which the system cannot escape any more. However, intuitively, one expects the approach to this state to occur on a time scale $\tau_{a c t} \sim q^{L}$ which is exponentially large in system size $L$ if parameters are chosen to represent the active phase of the thermodynamic limit. In the absorbing phase, both exact analytical results for $\lambda=D$ [8] and renormalization group results on diffusion-limited annihilation $(\alpha=0)$ [9] show that the approach to extinction is algebraic for the infinite system. For a finite system one can infer from these results a crossover time scale $\tau_{a b s} \sim L^{2}$ to exponential decay of the particle density.

Here we aim at obtaining information on the form of the phase transition line and on the dynamical and stationary behaviour of the system in various limiting cases involving fast diffusion of particles (or spins in terms of the spin-relaxation model). In Sec. III we derive a duality relation which maps the phase diagram onto itself in a non-trivial way. This duality is different from the domain-wall duality which maps the spin-relaxation dynamics to the BARW. On a self-dual line running across the phase diagram we obtain a relation between the (time-dependent) density expectation value for a halffilled random initial state and the survival probability of two single particles in an initially otherwise empty system.

In Sections III and IV we adopt the strategy of considering separately the fluctuations in the total number of particles from the spatial correlations within a configuration of a given fixed number of particles. By separating the hopping time scale from the branching and annihilation time scales one can then gain insight in the behaviour of the system in the absence of spatial correlations. In Sec. III we study the system in the fastdiffusion limit $D \rightarrow \infty$ of the BARW (2) (IIIA) and in its spin-relaxation formulation (1) in the dual limit of infinite spin-exchange rate $\alpha$. In these limiting cases all spatial correlations are washed out and one expects the PC transition to change into some other, mean-fieldtype phase transition. However, in contrast to a traditional mean-field approach, our treatment keeps track of the exact fluctuations of the total number of particles (or spins respectively). Our treatment is not an approximation, but yields rigorous results in these limiting cases for which we calculate the stationary density and density fluctuations (III A) and fluctuations of the magnetization (III B). Thus we are able to analyze to which extent the system deviates from mean-field behaviour and we also identify the exact phase transition point. In Sec. IV we investigate by similar means the dynamical behaviour of the finite system in the active region of the phase diagram. We show that for fast diffusion the relaxation to the absorbing state in a finite system is indeed exponentially slow, thus confirming the intuitive argument for the signature of the active region in a finite system.

In Section $\mathrm{V}$ we conclude with some final remarks.

\section{DUALITY RELATIONS}

We define the BARW in terms of a master equation for the probability $P(\eta ; t)$ of finding, at time $t$, a configuration $\eta$ of particles on a lattice of $L$ sites. Here $\eta=\{\eta(1), \eta(2), \ldots, \eta(L)\}$ where $\eta(x)=0,1$ are the integer-valued particle occupation numbers at site $x$. For definiteness we assume $L$ to be even. Using standard techniques [10] we express the time evolution given by the master equation in terms of a Hamiltonian $H$ of a one-dimensional quantum spin system (for a review see [11). The idea is to represent each of the possible particle configurations $\eta$ by a column vector $|\eta\rangle$ which together with the transposed vectors $\langle\eta|$ form an orthonormal basis of a vector space $X=\left(\mathbb{C}^{2}\right)^{\otimes L}$. One represents the probability distribution by a state vector $|P(t)\rangle=\sum_{\eta \in X} P(\eta ; t)|\eta\rangle$ and writes the master equation in the form

$$
\frac{d}{d t} P(\eta ; t)=-\langle\eta|H| P(t)\rangle
$$

where the off-diagonal matrix elements of $H$ are the (negative) transition rates between states and the diagonal entries are the inverse of the exponentially distributed life times of the states. A state at time $t^{\prime}=t_{0}+t$ is given in terms of an initial state at time $t_{0}$ by

$$
\left|P\left(t_{0}+t\right)\right\rangle=\mathrm{e}^{-H t}\left|P\left(t_{0}\right)\right\rangle .
$$

The expectation value $\rho_{k}(t)=\left\langle s\left|n_{k}\right| P(t)\right\rangle$ for the density at site $k$ is given by the projection operator $n_{k}$ which has value 1 if there is a particle at site $k$ and 0 otherwise. The summation vector $\langle s|=\sum_{\eta \in X}\langle\eta|$ performs the average over all possible final states of the stochastic time evolution. Below an initial distribution with $N$ particles placed on sites $k_{1}, \ldots, k_{N}$ is denoted by the column vectors $\left|k_{1}, \ldots, k_{N}\right\rangle$. The empty lattice is represented by the vector $|0\rangle$. The uncorrelated product distribution where on each lattice site the probability of finding a particle is equal to $1 / 2$, is given in terms of the transposed of the summation vector as $|1 / 2\rangle=\left\langle\left. s\right|^{T} /\left(2^{L}\right)\right.$.

To obtain the Hamiltonian for the time evolution of the BARW (2) we note that we can represent any twostate particle system as a spin system by identifying a particle (vacancy) on site $k$ with a spin-up (down) state on this site. This allows for a representation of $H$ in terms of Pauli matrices where $n_{k}=\left(1-\sigma_{k}^{z}\right) / 2$ projects on states with a particle on site $k$ and $v_{k}=1-n_{k}$ is the projector on vacancies. The off-diagonal matrices $s_{k}^{ \pm}=$ $\left(\sigma_{k}^{x} \pm i \sigma_{k}^{y}\right) / 2$ create $\left(s_{k}^{-}\right)$and annihilate $\left(s_{k}^{+}\right)$particles. We stress that in the present context the "spins" are just convenient labels for particle occupancies which are conceptually entirely unrelated to the spins of the spin relaxation model (11) which is treated below. Using this pseudospin formalism one finds

$$
\begin{aligned}
H= & \frac{1}{2} \sum_{k}\left\{D\left(n_{k} v_{k+1}+v_{k} n_{k+1}-s_{k}^{+} s_{k+1}^{-}-s_{k}^{-} s_{k+1}^{+}\right)\right. \\
& \left.+2 \lambda\left(n_{k} n_{k+1}-s_{k}^{+} s_{k+1}^{+}\right)+\alpha\left(1-\sigma_{k}^{x} \sigma_{k+1}^{x}\right) n_{k}\right\} .
\end{aligned}
$$


Each part of this stochastic Hamiltonian represents one of the elementary processes (2) and we may write

$$
H(D, \lambda, \alpha)=D H^{S E P}+\lambda H^{R S A}+\alpha H^{B A R W} .
$$

Here $H^{S E P}$ represents hopping of hard-core particles, i.e. the symmetric exclusion process [12], the pairannihilation process encoded in $H^{R S A}$ corresponds to random-sequential adsorption [13] and $H^{B A R W}$ describes a special equilibrium branching-annihilating random walk where pair annihilation requires the presence of another particle and hopping occurs only in pairs (see (2). For $\lambda=D$ and $\alpha=0$ this process reduces to the exactly solvable process of diffusion-limited pair annihilation (DLPA) 14. The time evolution conserves particle number modulo 2. Here we work only on the even subspace defined by the projector $(1+Q) / 2$ where $Q=(-1)^{N}=\prod_{k} \sigma_{k}^{z}$. The projection on the even sector of the uncorrelated initial state with a density $1 / 2$ is given by the vector $|1 / 2\rangle^{\text {even }}=(1 / 2)^{L-1}|s\rangle^{\text {even }}$.

Within the same framework the stochastic Hamiltonian for the spin-flip process (11) can be written in terms of Pauli matrices as follows

$$
\begin{aligned}
H^{S F}= & \frac{1}{4} \sum_{k}\left[\left(1-\sigma_{k}^{x}\right) w_{k}(D, \lambda)+\right. \\
& \left.+\alpha\left(1-\sigma_{k}^{x} \sigma_{k+1}^{x}\right)\left(1-\sigma_{k}^{z} \sigma_{k+1}^{z}\right)\right]
\end{aligned}
$$

with the generalized Glauber spin flip rates encoded in $w_{k}(D, \lambda)=\left(2-\sigma_{k-1}^{z} \sigma_{k}^{z}-\sigma_{k}^{z} \sigma_{k+1}^{z}\right)(D+\lambda+(\lambda-$ $\left.D) \sigma_{k-1}^{z} \sigma_{k+1}^{z}\right) / 2$. For this process the spins represent the actual spin configurations of the spin-relaxation process. We note that the usual zero-temperature Glauber dynamics - equivalent to DLPA in particle language correspond to $\lambda=D, \alpha=0$. For this model the domain wall correspondence [7] between the BARW and the spin-flip process can be rigorously derived as a similarity transformation on the level of the quantum Hamiltonian description. There exists a transformation $\mathcal{B}$ such that $H^{S F}=\mathcal{B H B}^{-1}$ 15. The generalization $\lambda=D, \alpha>0$ corresponds to the exactly solvable process introduced in Ref. [8].

Consider now the transformation $\mathcal{D}_{ \pm}$which is, for the even particle sector, defined by

$$
\mathcal{D}_{+}=\gamma_{1} \gamma_{2} \ldots \gamma_{2 L-1}
$$

where

$$
\begin{aligned}
\gamma_{2 k-1} & =\frac{1}{2}\left[(1+i) \sigma_{k}^{z}-(1-i)\right] \\
\gamma_{2 k} & =\frac{1}{2}\left[(1+i) \sigma_{k}^{x} \sigma_{k+1}^{x}-(1-i)\right] .
\end{aligned}
$$

and defined by $\mathcal{D}_{-}=-\mathcal{D}_{+} \sigma_{L}^{x}$ for the odd particle sector [16, 17. $\mathcal{D}_{ \pm}$is unitary and transforms Pauli matrices as follows:

$$
\begin{aligned}
\mathcal{D}_{ \pm}^{-1} \sigma_{k}^{x} \sigma_{k+1}^{x} \mathcal{D}_{ \pm} & = \begin{cases}\sigma_{k}^{z} & k \neq L \\
Q \sigma_{L}^{z} & k=L\end{cases} \\
\mathcal{D}_{ \pm}^{-1} \sigma_{k+1}^{z} \mathcal{D}_{ \pm} & = \begin{cases}\sigma_{k}^{x} \sigma_{k+1}^{x} & k \neq L \\
\pm Q \sigma_{L}^{x} \sigma_{1}^{1} & k=L\end{cases}
\end{aligned}
$$

In Ref. 18 it was observed that this transformation maps $H^{D L P A}$, obtained from $H$ by setting $\lambda=D$ and $\alpha=0$, onto its transposed, $H^{D L P A}=\mathcal{D}\left(H^{D L P A}\right)^{T} \mathcal{D}^{-1}$ and thus generates a set of relations between various expectation values [19]. Here we go further and apply this transformation to the Hamiltonian $H=H(D, \lambda, \alpha)$ (5) and transpose the operator which results from the transformation. Using (11), (12) we find

$$
\begin{aligned}
H^{S E P} & \rightarrow H^{B A R W}, \\
H^{B A R W} & \rightarrow H^{S E P}, \\
H^{R S A} & \rightarrow H^{R S A}+H^{S E P}-H^{B A R W}
\end{aligned}
$$

and hence the relations

$$
\tilde{H}=\lambda H^{R S A}+(\lambda+\alpha) H^{S E P}+(D-\lambda) H^{B A R W} .
$$

which has the same form as the original Hamiltonian (6), but with rates

$$
\begin{aligned}
\tilde{\lambda} & =\lambda, \\
\tilde{D} & =\lambda+\alpha, \\
\tilde{\alpha} & =D-\lambda .
\end{aligned}
$$

The transformation (13) is a duality transformation, we obtain the identity transformation if we apply the transformation twice.

On the mean-field phase transition line $\lambda=D$ [20] the system is exactly solvable [8] and belongs in its entirety to the inactive phase. Hence the whole region $\lambda>D$ is in the inactive phase. Duality maps the interesting region $\lambda \leq D$ which contains the phase transition line nontrivially onto itself. Thus duality can be used to relate physical quantities at different points of the parameter space. This region contains a self-dual line

$$
D=\lambda+\alpha
$$

in which every point maps onto itself. In the notation of Ref. [3] $D=p_{r w}=\Gamma^{-1}(1-\delta), \lambda=p_{a n}=\Gamma^{-1}(1+\delta)$ and $\alpha=2 p_{e x}=2\left(1-2 \Gamma^{-1}\right)$ is normalized such that $p_{e x}+$ $p_{r w}+p_{a n}=1$. With a parametrization in terms of $\delta$ and $p_{e x}$ the dual rates are given by $\tilde{\delta}=-2 p_{e x} /\left[1+p_{e x}+\delta(1-\right.$ $\left.\left.p_{e x}\right)\right]$ and $\tilde{p_{e x}}=-\delta\left(1-p_{e x}\right) /\left[\delta\left(1-p_{e x}\right)+2\left(1+p_{e x}\right)\right]$. The self-dual line is given by the relation $\delta=-2 p_{\text {ex }} /\left(1-p_{\text {ex }}\right)$.

The duality transformation not only maps the phase-diagram onto itself, but also generates relations between time-dependent expectation values. Consider the expectation value of the density $\rho_{k}(t)=$ $\left\langle\left. s\right|^{\text {even }} n_{k} e^{-H t} \mid 1 / 2\right\rangle^{\text {even }}$, where $|1 / 2\rangle^{\text {even }}$ is a random initial state with density $1 / 2$, projected over the even sector. This expectation value is defined at the point $(D, \lambda, \alpha)$ of the parameter space of the Hamiltonian. It is straightforward to verify the relations

$$
\begin{aligned}
\mathcal{D}^{-1}|s\rangle^{\text {even }} & =-i(i-1)^{L-1}|0\rangle \\
\left\langle 1 /\left.2\right|^{\text {even }} \mathcal{D}\right. & =i(-i-1)^{L-1}\langle 0| / 2^{L-1}
\end{aligned}
$$


So if we use these rules of transformation and the rules of transformation for the Pauli matrices, given by (11), (12), we can write the expectation value for the density in the even sector the form

$$
\begin{aligned}
\left\langle s\left|n_{k} e^{-H t}\right| 1 / 2\right\rangle^{\text {even }} & =\frac{1}{2}\left\langle 0\left|e^{-\tilde{H} t}\left(1-\sigma_{k-1}^{x} \sigma_{k}^{x}\right)\right| 0\right\rangle \\
& =\frac{1}{2}\left(1-\left\langle 0\left|e^{-\tilde{H} t}\right| k, k+1\right\rangle\right)
\end{aligned}
$$

where we have used (13) and the fact that the expectation value for the density $\rho_{k}(t)$ is a real number. The transformed initial state is a superposition of the steady state (the empty lattice) and the two-particle state with particles at sites $k, k+1$. The quantity in the right hand side of equation (17) is one-half times the probability that the state with two particles initially placed at sites $k$ and $k+1$ has not decayed at time $t$ to the empty state, measured with the transformed rates $\tilde{\lambda}, \tilde{D}, \tilde{\alpha}(14)$.

This is a specific result for the time-dependent density starting from a random initial state with density $1 / 2$. More general transformation properties of timedependent correlation functions can be obtained following the strategy of Ref. [18]. We conclude this section by pointing out that analogous enantiodromy relations can be derived for a discrete-time version of the process which corresponds to a sublattice parallel updating scheme rather than the random sequential updating represented by the stochastic Hamiltonians (5), (7). Such a parallel updating scheme (which we expect to retain all the universal features of the model) consists of four steps. In a first updating sweep, update all spins on the even sublattice in parallel according to the generalized Glauber rules, but with the rates $\lambda, D$ now taken as actual probabilities. In a second step one updates the odd sublattice. In a third step one applies a sublattice parallel pair-updating scheme to implement the Kawasaki spinexchange process with probability $\alpha / 2$ : In a first round one divides the lattice into even/odd pairs $(2 k, 2 k+1)$ and exchanges spins within each pair with probability $\alpha / 2$. Finally one updates with the odd/even pairs. This completes a full updating cycle. The stochastic time evolution of this process may be written in terms of the transfer matrix

$$
T^{S F}=T_{\text {odd }}^{K}(\alpha) T_{\text {even }}^{K}(\alpha) T_{\text {odd }}^{G}(\lambda, D) T_{\text {even }}^{G}(\lambda, D)
$$

where

$$
T_{\text {even }}^{G}(\lambda, D)=\prod_{k=1}^{L / 2}\left[1-\left(1-\sigma_{k}^{x}\right) w_{k}(D, \lambda) / 4\right]
$$

and an analogous expression for $T_{\text {odd }}^{K}$. The spin exchange transfer matrix $T_{o d d}^{K} T_{\text {even }}^{K}$ is the well-known transfer matrix of the six-vertex model [21] defined on a diagonal square lattice. One has

$$
T_{\text {even }}^{K}=\prod_{k=1}^{L / 2}\left[1-\alpha\left(1-\sigma_{2 k}^{x} \sigma_{2 k+1}^{x}\right)\left(1-\sigma_{2 k}^{z} \sigma_{2 k+1}^{z}\right) / 4\right] .
$$

The transfer matrix for the related BARW model can be obtained by applying the similarity transformation $\mathcal{B}$ of Ref. [15]. One can then derive a duality relation in the way described above. The transformed process has the same elementary transitions, but with a different updating sequence.

\section{PHASE TRANSITION FOR FAST DIFFUSION}

It is intuitively clear that the $\mathrm{PC}$ phase transition in the system originates in the complicated structure of the density correlations which are build up by the competing processes of branching and annihilation. For a better understanding, consider first $\lambda=0$. This reduced process includes (besides diffusion) branching $A \rightarrow 3 A$ and conditional pair annihilation $3 A \rightarrow A$ which both require the presence of a surviving particles to take place. As a result, there are two stationary distributions: the empty lattice, and the random distribution where each particle configuration is equally likely. Since there is no transition channel from the occupied lattice to the empty lattice, the system is in the active phase.

On the other hand, for $\alpha=0$ the system is in the absorbing phase, the only stationary distribution is the empty lattice. We conclude that the unconstrained pair annihilation process $2 A \rightarrow 0$ with rate $\lambda$ is responsible for the phase transition to take place.

This scenario is captured in a simple mean-field approach. The exact equations of motion for the expected particle number $\langle N(t)\rangle$ reads

$$
\frac{d}{d t}\langle N(t)\rangle=\sum_{i}\left[\alpha\left\langle n_{i}(t)\right\rangle-2(\alpha+\lambda)\left\langle n_{i}(t) n_{i+1}(t)\right\rangle\right] \text {. }
$$

Replacing the correlators by the product of the density $\rho(t)=\left\langle n_{i}(t)\right\rangle$ yields the mean-field equation

$$
\frac{d}{d t}\langle N(t)\rangle=\alpha\langle N(t)\rangle-\frac{2(\alpha+\lambda)}{L}\langle N(t)\rangle^{2}
$$

with the stationary mean-field solution for the active phase

$$
\langle N\rangle_{m f}^{*}=\frac{\alpha}{2(\alpha+\lambda)} L
$$

Since each lattice site can take only one particle and therefore $n_{i}=0,1$, one can use $n_{i}^{2}=n_{i}$ to show that the mean-field fluctuations $\Delta_{m f}^{*}=\left\langle N^{2}\right\rangle_{m f}^{*}-\left(\langle N\rangle_{m f}^{*}\right)^{2}$ around the mean are given in terms of the density $\rho_{m f}^{*}=$ $\langle N\rangle_{m f}^{*} / L$ by

$$
\Delta_{m f}^{*}=\rho_{m f}^{*}\left(1-\rho_{m f}^{*}\right) L .
$$

We conclude that the mean-field phase transition point is given by $\alpha / \lambda=0$, consistent with the considerations 
above. By duality (14) we also recover the mean-field phase transition line of Ref. [20].

There are several questions that we want to address in this context. The first is the form of the exact phase transition line if both $\alpha$ and $\lambda$ are very small compared to the diffusion rate $D$. The second question is the nature of the phase transition in this limit. If $D \gg \alpha, \lambda$ the spatial correlations build up by the annihilation/branching process are wiped out very quickly by diffusive mixing, leaving a transition which we cannot expect to be a PC transition anymore. Finally, in the next section we study the crossover time scales on which a large, but finite system reaches the absorbing state.

\section{A. Phase transition in the BARW}

To tackle these questions we observe that for fast diffusion the process simplifies dramatically: In the absence of spatial correlations the state of the system is fully characterized by the total particle number $N$. For fixed $N$, each particle configuration occurs with equal probability $N !(L-N) ! /(L)$ ! which is just the inverse of the number of possibilities of placing $N$ particles on a lattice of $L$ sites. As a result, the dynamics reduce to a random walk on the integer set $0,2,4, \ldots, 2 K, \ldots, L$ of total particle number $N=2 K$. Thus we may represent the dynamics as a random walk on a one-dimensional lattice of $L / 2+1$ sites, where the position of the random walker marks the number of particles of the BARW process and 0, representing the empty lattice, is an absorbing point. It remains only to calculate the hopping rates $r_{N}$ and $\ell_{N}$ from site $N$ to the right $(N+2)$ and left $(N-2)$ respectively. The state of the system is then given by the solution of the master equation

$$
\begin{aligned}
\frac{d}{d t} P_{N}(t)= & r_{N-2} P_{N-2}(t)+\ell_{N+2} P_{N+2}(t) \\
& -\left(r_{N}+\ell_{N}\right) P_{N}(t)
\end{aligned}
$$

for the probability of finding $N$ particles in the system. The average particle number is given by $\langle N(t)\rangle=$ $\sum_{N} N P_{N}(t) 22$.

By counting the number of possibilities of finding two vacancies on neighbouring sites of an occupied site in a random state of $N$ particles one readily finds

$$
r_{N}=\frac{\alpha}{2} \frac{N(L-N)(L-N-1)}{(L-1)(L-2)}
$$

as contribution from the branching process with rate $\alpha / 2$. An analogous consideration gives

$$
\ell_{N}=\frac{1}{2}\left[\frac{\alpha N(N-1)(N-2)}{(L-1)(L-2)}+\frac{2 \lambda N(N-1)}{(L-1)}\right]
$$

as the contribution from the annihilation processes $3 A \rightarrow$ $A$ and $2 A \rightarrow 0$ respectively.
These rates represent a biased random walk which in the thermodynamic limit $L \rightarrow \infty$ and for $N$ fixed reduces to a directed random walk in positive direction with increasing hopping rate $r_{N}=\alpha N / 2$. Since for $D \rightarrow \infty$ the branching process is not diffusion-limited, the particle number increases exponentially in time, $\langle N(t)\rangle=$ $\langle N(0)\rangle e^{\alpha t}$. Thus for any $\alpha>0$ the system is in the active phase, i.e., the phase transition is at $\alpha=0$ which is consistent with the mean-field result (23). For a large, but finite system with a small initial number of particles one expects a slowing down of the exponential growth when a finite density is reached, i.e. on a time scale of the order $\ln (L) / \alpha$. Ultimately, though, the finite system will reach, by a rare fluctuation in the number of particles, the absorbing empty lattice. This second crossover time to absorption is discussed in the next section.

To study the stationary behaviour $d /(d t) P_{N}(t)=0$ of the system we rescale the lattice to unit length and expand the r.h.s. of the master equation 25 in a Taylor series in the lattice spacing $1 / L$. Setting $x=N / L$ and keeping the leading order term yields the equation $c=$ $2\left(l_{x}-r_{x}\right) P_{x}^{*}$. The integrability condition $\int_{0}^{1} d x P_{x}^{*}=1$ on the stationary probability distribution requires the integration constant $c$ to vanish. The resulting equation has the solution $P_{x}^{*}=\delta(x)$, corresponding to the absorbing state. The only other integrable solution on the interval $[0,1]$ is the delta-function $P_{x}^{*}=\delta\left(x-\rho^{*}\right)$ with

$$
\rho^{*}=\frac{\alpha}{2(\alpha+\lambda)}
$$

This gives the exact stationary density $\rho^{*}$ of the active phase which, not very surprisingly, coincides with the mean-field value (23).

To determine whether the system in the infinitediffusion limit actually is a mean-field system we investigate the fluctuations around the mean (28). The mean field result (24) requires studying the fluctuations on a length scale of order $y=\sqrt{L}\left(x-\rho^{*}\right)$. Keeping in the Taylor expansion of the master equation around $x=\rho^{*}$ all terms to this order gives the ordinary differential equation

$$
\frac{d}{d y} P_{y}^{*}=-\frac{y}{2 \rho^{*}\left(1-\rho^{*}\right)^{2}} P_{y}^{*}
$$

The solution of this equation is a Gaussian which gives the exact fluctuations in the particle number

$$
\Delta^{*}=2 \rho^{*}\left(1-\rho^{*}\right)^{2} L
$$

Except for $\lambda=0\left(\rho^{*}=1 / 2\right)$ this expression is in disagreement with the mean-field result (24), indicating a non-trivial effect of the unconstrained pair-annihilation process even in the fast diffusion limit. We conclude that the system undergoes a mean-field transition, but with fluctuations in the particle number which deviate from those predicted by mean-field. 


\section{B. Spin-relaxation formulation}

We consider the system in the dual limit $\alpha \rightarrow \infty$ where we can study the phase transition between the active phase and the absorbing phase in terms of the dimensionless variable $u=(D-\lambda) /(D+\lambda)$. In the spin-relaxation picture this is the limit of fast Kawasaki spin-exchange where the system is spatially uncorrelated and hence completely characterized by the total magnetization $M=\sum_{k} \sigma_{k}^{z} / 2$. The dynamics of the process (1) reduce in this limit to a random walk in the magnetization variable $M$, ranging from $-L / 2$ to $L / 2$. The master equation reads

$$
\begin{aligned}
\frac{d}{d t} P_{M}(t)= & r_{M} P_{M-1}(t)+\ell_{M+1} P_{M+1}(t) \\
& -\left(r_{M}+\ell_{M}\right) P_{M}(t) .
\end{aligned}
$$

The transition rates for this random walk with absorbing boundaries at $M= \pm L / 2$ are readily calculated as

$$
\begin{aligned}
& r_{M}=\frac{D+\lambda}{2 L-2}\left(1-\frac{2 M u}{L-2}\right)\left(\frac{L^{2}}{4}-M^{2}\right) \\
& \ell_{M}=\frac{D+\lambda}{2 L-2}\left(1+\frac{2 M u}{L-2}\right)\left(\frac{L^{2}}{4}-M^{2}\right) .
\end{aligned}
$$

We find a bias towards to the boundaries $M= \pm L / 2$, i.e., the fully magnetized absorbing states with all spins up or down resp., for $u<0$. For $u>0$ the system is biased to the center, corresponding to the active phase.

For an initial state which is symmetric under spin-flip $s_{i} \rightarrow-s_{i}$ the mean $\langle M\rangle$ vanishes for all times in both the active and absorbing phase and hence is not suitable to characterize the system. For the same reason an naive mean-field approach by setting $\left\langle\sigma_{i}^{z}(t) \sigma_{j}^{z}(t)\right\rangle=$ $\left\langle\sigma_{i}^{z}(t)\right\rangle\left\langle\sigma_{j}^{z}(t)\right\rangle$ would not give any information on the dynamics of the spin-fluctuations. The quantity that characterizes the phase transition are the fluctuations $\left\langle M^{2}\right\rangle=\sum_{M} M^{2} P_{M}(t)$ in the magnetization, i.e. the mean-square displacement of the random walk. In the active regime this quantity is proportional to the system size whereas in an ordered state $\left\langle M^{2}\right\rangle \sim L^{2}$.

First consider the phase transition point $u=0$. From the considerations above we know that the stationary state is inactive. The only question of interest is the approach to stationarity from some random initial state. From (31) one obtains $d /(d t)\left\langle M^{2}\right\rangle=$ $2 \lambda\left(L^{2} / 4-\left\langle M^{2}\right\rangle\right) /(L-1)$ which is readily solved by

$$
\left\langle M^{2}(t)\right\rangle=L^{2} / 4+\left(\left\langle M^{2}(0)\right\rangle-L^{2} / 4\right) e^{-2 \lambda t /(L-1)} .
$$

The approach to the stationary value is exponential on a time scale

$$
\tau=\frac{L-1}{2 \lambda}
$$

For large system size and initial times $\tau \ll L$, the fluctuations in the magnetization grow linearly in time.
For $u \neq 0$ the equations of motion for the moments $\left\langle M^{2 k}\right\rangle$ are too complicated for direct analysis. We define $\hat{M}=M / \sqrt{L}$ and study only the thermodynamic limit. Using the master equation (31) the stationarity condition $d /(d t)\left\langle\hat{M}^{2 k}\right\rangle=0$ for the moments of $\hat{M}$ yields the recursion relation

$$
\left\langle\hat{M}^{2 k}\right\rangle=(2 k-1)\left\langle\hat{M}^{2 k-2}\right\rangle /(4 u)
$$

which shows that the stationary distribution in the active phase $u>0$ is Gaussian with variance $1 /(4 u)$ :

$$
P^{*}(\hat{M})=\sqrt{\frac{2 u}{\pi}} e^{-2 u \hat{M}^{2}} .
$$

This yields the final result

$$
\left\langle\hat{M}^{2}\right\rangle=\left\{\begin{array}{cc}
1 /(4 u) & u>0 \\
\infty & u \leq 0
\end{array}\right.
$$

All other stationary moments in the active regime follow from the Gaussian nature (37) of the statistics. We read off a critical exponent $\kappa=1$ for the divergence of $\left\langle\hat{M}^{2}\right\rangle$ with $u$ as the system approaches the critical point $u=0$.

\section{RELAXATIONAL BEHAVIOUR IN FINITE SYSTEMS}

The exact solution [8] for the dynamics of the spin-spin correlation function on the line $\lambda=D$ implies a crossover time $\tau \sim L^{2}$ from a power law relaxation to exponential relaxation to the absorbing state. One then expects this to hold throughout the inactive phase.

On the other hand, any finite system has only one stationary state, which is the empty lattice in particle language, corresponding to the magnetically ordered states with all spins up or all spins down. It is therefore of interest to study the relaxation towards this state in that region of parameter space that constitutes the active phase of the infinite system.

The precise location of the phase transition line is not known, but we know that the line $\lambda=0$ (no unrestricted pair annihilation) belongs to the active phase and thus we may get some insight by studying the system in the immediate neighbourhood $\lambda \ll \alpha, D$ of this line. To this end we adopt a similar strategy as in the previous section by assuming $\lambda$ to be so small that the system had sufficient time to relax to its $\lambda=0$ stationary distribution between two successive pair-annihilation events. This limiting procedure can be made rigorous by taking $\alpha \rightarrow \infty$ and keeping $\lambda, D$ fixed.

In this limit the system reduces effectively to a twostate system, i.e. the system is completely characterized by stating whether the system is empty (state $|0\rangle$ ) or not (which we denote by $|1\rangle$ ). The latter state represents the stationary distribution of the system with $\lambda=0$ in which, because of detailed balance for this reduced process, all 
states with an even, non-zero number of particles have equal probability $p=1 /\left[2^{(L-1)}-1\right]$. The transition rates between these two states characterizing the system are then trivial to work out: The transition from $|0\rangle$ to $|1\rangle$ is zero because $|0\rangle$ is an absorbing state. On the other hand, counting the number of states represented by $|1\rangle$ for which a pair annihilation event leads to the empty lattice yields a transition rate

$$
1 / \tau_{a c t}=(\lambda L) /\left[2^{(L-1)}-1\right]
$$

for transitions from state 1 to state 0 . Hence, at time $t$ the system is in the absorbing state with probability $P_{0}(t)=1-e^{-t / \tau_{a c t}}$ and in each non-empty state with probability $P_{1}(t)=e^{-t / \tau_{a c t}} /\left[2^{(L-1)}-1\right]$.

For the particle density this behaviour implies the exact result

$$
\rho(t)=\frac{e^{-t / \tau_{a c t}}}{2-(1 / 2)^{L}} .
$$

Because of the fast intermediate relaxation to the equilibrium state of the $\alpha=\infty$ process the density and the density-correlations have no spatial dependence and the diffusion rate does not enter. The crossover time $\tau_{a c t}$ for reaching the absorbing state in the active region of the phase diagram for the infinite system ( small $\lambda$ ) diverges exponentially in system size.

Finally, we study the dynamical behaviour of the system for large, but finite $L$ in the limit of fast diffusion dicussed in Sec. III A. We recall relation (17) which relates the decay of the particle density to the survival probability of two neighouring particles in an empty lattice. This quantity can be interpreted as a first-passage-time distribution for two annihilating and branching random walkers: When two random walkers in an empty lattice annihilate for the first time, the dynamics stop. Therefore the density decay equals one half this first-passage-time distribution and the mean-first-passage-time (MFPT)

$$
\begin{aligned}
\tau & =\int_{0}^{\infty} d t\left\langle 0\left|e^{-H t}\right| k, k+1\right\rangle \\
& =\lim _{c \rightarrow 0}\left\langle 0\left|(H+c)^{-1}\right| k, k+1\right\rangle
\end{aligned}
$$

gives the crossover time scale on which the system reaches the absorbing state.

This quantity can be evaluated numerically for any point in parameter space by inverting the time evolution operator $c+H$ for finite system size, then taking the matrix element (41) and finally calculating the limit $c \rightarrow 0$. For an analytical treatment for large $D$ we note that the MFPT from some site $k$ to an absorbing site $k=0$ for a general random walk with nearest neighbour hops on $L+1$ sites can be expressed in terms of the hopping rates 23. In the mapping of the BARW to the random walk the MFPT $\tau$ is equal to the MFPT of the random walker starting at site 2 . With the hopping rates (26), (27) we find after some rearrangement of terms

$$
\tau=\frac{1}{\lambda L} \sum_{k=0}^{L / 2-1} c_{k}
$$

with

$$
c_{k}=\frac{L ! k ! \Gamma(\lambda(L-2) / \alpha+1)}{(2 k+2) !(L-2 k-2) ! \Gamma(\lambda(L-2) / \alpha+k+1)} .
$$

We note first that in the limit $\alpha \rightarrow \infty$ the MFPT coincides with the relaxation time (39), as expected from duality. To study the asymptotic behaviour of $\tau$ for finite $\alpha$ (active phase) we determine the value $k_{0}$ for which $c_{k}$ gives the largest contribution to sum on the r.h.s. of (42). We find $k_{0}=\rho^{*} L / 2$ with the stationary density given by (28). Using the Stirling formula for the Gamma-function and expanding $c_{k}$ around $k_{0}$ yields for non-vanishing density $\rho^{*}$ the asymptotic form of the crossover time

$$
\tau_{a c t} \sim \frac{1}{\lambda}\left[\frac{\left(1-2 \rho^{*}\right)^{\left(1-2 \rho^{*}\right) /\left(2 \rho^{*}\right)}}{\left(1-\rho^{*}\right)^{\left(1-\rho^{*}\right) /\left(\rho^{*}\right)}}\right]^{L}
$$

up to subleading power-law corrections in system size. Therefore, in the active region of the phase diagram the crossover to absorption in a finite system takes place on a time scale which is exponentially large in system size, with a density-dependent amplitude.

For $\rho^{*}=0$, i.e. in the absorbing phase, the MFPT can be read off directly from 42 , (43), since only the term with $k=0$ contributes. One finds

$$
\tau_{a b s}=\frac{(L-1)}{2 \lambda}
$$

This power law differs from the crossover behaviour $\tau_{a b s} \sim L^{2} / D$ for finite diffusion constant D. The MFPT for this point in parameter space coincides with the relaxation time (35) in the dual point.

\section{FINAL REMARKS}

The duality relation (13) divides the parameter space into two distinct regions separated by the self-dual line (15). Both regions are mapped onto each other and hence have the same physical properties. This is of practical usefulness for a numerical survey of the system and for the determination of the phase transition line since only part of the parameter space needs to be investigated.

In particular, the line $\alpha=0$ maps onto the line $D=\lambda$ and the fast-diffusion limit to the limit $\alpha \rightarrow \infty$. The observation that for large $D$ any small $\alpha$ brings the system into the active phase translates into a phase transition at $D=\lambda$ in the limit $\alpha \rightarrow \infty$. This is a new result which definitely clarifies the unresolved issue of the location of the phase transition line for large $\alpha$. Numerical analysis of the model for large rates $\alpha$ is reported to be very difficult [3,20]. Our exact result confirms the conjecture 
of Ref. [3] on the location of the phase transition point in this numerically untractable limit. In the $\delta-p_{e x}$ phase diagram of Ref. [3] the limit $\alpha \rightarrow \infty$ corresponds to $p_{e x} \rightarrow 1$ and the phase transition point $D=\lambda$ corresponds to $\delta=0$. The dual limit $D \rightarrow \infty$ covers the neighbourhood of the point $\delta=-1, p_{e x}=0$. Our result translates into an infinite slope of the phase transition line in this representation at this point. In the active phase of this region the exact stationary particle number distribution is Gaussian with a stationary density $\rho^{*}$ given by the mean-field value (23). The density fluctuations (30) deviate from the mean-field result (24) by a factor $2\left(1-\rho^{*}\right)$.

On the self-dual line we find from (17) that the density expectation value $\rho_{k}(t)$ equals one-half the survival probability at time $t$ of two particles placed initially at two neighbouring sites in an otherwise empty lattice. Hence the phase transition from the absorbing phase to the active phase may be rephrased as a mean-first-passage-time (MFPT) transition for random walkers which branch and annihilate. We expect the MFPT in a finite system to change from a power-law divergence (in system size) to an exponential divergence not only at infinite diffusion rate (Sec. IV]), but also for finite $D$. Thus this numerically accessible quantity provides an alternative way of determining and characterizing the PC phase transition.

\section{ACKNOWLEDGEMENTS}

We thank J. Cardy, N. Menyhárd, Z. Rácz and U. Täuber for useful discussions. G.M.S. thanks the Institute for Theoretical Physics of the Eötvös University for hospitality and partial support (Grant OTKA OTKA T 019451).

[1] T. E. Harris, Ann. Prob. 2, 969 (1974); H. Takayasu and A. Yu. Tretyakov 68, 3060 (1992); I. Jensen, Phys. Rev E 47, R1 (1993), Phys. Rev. Lett. 70, 1465 (1993).

[2] P. Grassberger, F. Krause and T. von der Twer, J. Phys. A 17, L105 (1984); P. Grassberger, J. Phys. A 22, L1103 (1989); M. H. Kim and H. Park, Phys. Rev. Lett. 73, 2579 (1994); I. Jensen, J. Phys. A 26, 3921 (1993), Phys. Rev. E 50, 3623 (1994); D. ben-Avraham, F. Leyvraz and S. Redner, Phys. Rev. E 50, 1843 (1994). Some of the references consider BARW's with both even and odd number of offsprings.

[3] N. Menyhárd, J. Phys. A 27, 6139 (1994).

[4] J. Cardy and U. Täuber, Phys. Rev. Lett. 77, 4780 (1996); J. Stat. Phys. 90 (1998) (in press).

[5] R. J. Glauber, Math. Phys. 4, 294 (1963).

[6] K. Kawasaki, Phys. Rev. 145, 224 (1966).

[7] Z. Rácz, Phys. Rev. Lett. 55, 1707 (1985).
[8] M. Droz, Z. Rácz and J. Schmidt, Phys. Rev. A 39, 2141 (1989).

[9] B. P. Lee, J. Phys. A 27, 2633 (1994).

[10] E. Siggia, Phys. Rev. B 16, 2319 (1977); S. Sandow and S. Trimper, Europhys. Lett. 21, 799 (1993); F. C. Alcaraz, M. Droz, M. Henkel and V. Rittenberg, Ann. Phys. (N.Y.) 230, 250 (1994); G. M. Schütz, J. Stat. Phys. 79, 243 (1995).

[11] G. M. Schütz, Integrable Reaction-Diffusion Processes and Quantum Spin Chains (to be published).

[12] F. Spitzer, Adv. Math. 5, 246 (1970).

[13] J. W. Evans, in Nonequilibrium Statistical Mechanics in One Dimension, ed. V. Privman (Cambrige University Press, Cambridge UK, 1996).

[14] D. C. Torney and H. M. McConnell, J. Phys. Chem. 87, 1941 (1983).

[15] J. E. Santos J. Phys. A 30, 3249 (1997).

[16] D. Levy, Phys. Rev. Lett. 67, 1971 (1991).

[17] G. Schütz J. Phys. A 26, 4555 (1993).

[18] G. M. Schütz, Z. Phys. B 104, 583 (1997); G. M. Schütz and K. Mussawisade, Phys. Rev. E 57 (1998) (in press).

[19] Such a relationship between a stochastic processes is called an enantiodromy relation, as opposed to a similarity transformation like the domain-wall duality which relates one stochastic Hamiltonian to another, rather than to its transposed.

[20] N. Menyhárd and G. Ódor J. Phys. A 28, 4505 (1995).

[21] D. Kandel, E. Domany and B. Nienhuis, J. Phys. A 23, L755 (1990).

[22] This and the other limits of fast rates discussed in this paper can be treated rigorously by taking the limit of large rates in the formal solution 4 of the master equation, see 111 for details.

[23] G. H. Weiss, J. Stat. Phys. 24, 587 (1981); K. P. N. Murthy and K. W. Kehr, Phys. Rev. A 40, 2082 (1989), and erratum in Phys. Rev. A 41, 1160 (1990). 